\title{
The solar plasma conditions in the source regions of two explosive events
}

\author{
J. E. Mendoza-Torres ${ }^{1}$, K. Wilhelm ${ }^{2}$, and A. Lara ${ }^{3}$ \\ ${ }^{1}$ Instituto Nacional de Astrofísica, Optica y Electrónica, Apartado Postal 51 y 216, Puebla, Pue., Z.P. 72000, México \\ e-mail: mend@inaoep.mx \\ 2 Max-Planck-Institut für Sonnensystemforschung, 37191 Katlenburg-Lindau, Germany \\ 3 Instituto de Geofísica, UNAM, México
}

Received 11 October 2006 / Accepted 7 November 2008

\begin{abstract}
We analyze the ultraviolet emission during two explosive events (EEI and EEII) recorded by SUMER aboard SOHO in the wavelength range from $749 \AA$ to $789 \AA$. The events occurred near the centre of the solar disk on 17 November 1996 . An attempt is made to separate the emission of the primary energy release from that of the accelerated plasma. We want to establish the characteristics of the sources, in particular, how large they are and how they are spatially distributed with respect to each other. Gaussian fits were calculated to estimate the radiances of the brightest emissions in our wavelength range for the core of the lines and their blue and red wings. The maximum of each Gaussian approximation was used to define the spatial distributions of the components. The electron densities were obtained with the help of the OV line ratio. The relationship between the densities and the spatial distribution of the radiances is then analyzed. The distributions of EEI are more extended than those of EEII, probably due to the different geometries of the observations. In both events, there is an offset in the locations of red and blue components but the offset is greater at EEI than at EEII. The Doppler velocities of the components of EEI were smaller than those of EEII. Also, in both events the density of the red component attained the highest values $\left(\geq 4 \times 10^{10} \mathrm{~cm}^{-3}\right)$. In EEI the electron density distributions of the three components were considerably narrower than the radiance distributions. The peak densities were observed centred with respect to the radiance distributions, suggesting that the sources of the first energy release of this event were near these locations. Both the electron density and the radiance distributions of EEI suggest a jet direction close to the line of sight.
\end{abstract}

Key words. Sun: transition region - Sun: activity - Sun: UV radiation

\section{Introduction}

An outstanding problem of modern astrophysics is the heating of the solar corona. The solar transition region, where the temperature increases from chromospheric to coronal levels, seems to play a fundamental role in hypotheses of heating processes. A mechanical energy transport is believed to be an indispensible ingredient (see, e.g. Axford 1985; Esser et al. 1987; Axford \& McKenzie 1992). In view of the thin transition region, detailed studies of small-scale events are required to further our understanding of such processes. Explosive events (EE) could be particularly important, because of their high occurrence rate over the whole Sun, although it has been established that their particle energy alone is not sufficient, and the magnetic wave energy has to be considered as well.

The physical conditions at the site of the energy release in an EE are not well known. Theoretical and observational results seem to indicate that EEs are caused by magnetic reconnection in a scenario where a current sheet and bipolar jets are formed. Observations of strong, Doppler-shifted emission in the wings of ultraviolet spectral lines during EE support this idea (Innes et al. 1997; Chae et al. 1998; Landi et al. 2000; Mendoza-Torres et al. 2005). It is thus necessary to evaluate the parameters of both plasmas, i.e. at the source and the accelerated plasma. This knowledge is essential to test EE models and to decide whether the total energy released is a significant contribution to the coronal heating process or not. It is also required to distinguish the emission that stems from the primary release of energy of an EE from that produced around it (by secondary processes). In spite of the fact that many EEs have been observed by now, there are still many open questions concerning the different plasmas produced during an EE.

Profiles of spectral lines emitted by highly-ionized ions provide a tool to study these plasmas. They contain information on line-of-sight (LOS) turbulent velocities and bulk motions. Turbulent velocities lead to line broadening, indicative of the effective ion temperature, $T_{i, \text { eff }}$, and bulk motions result in line shift. However, the line profiles may lead to some ambiguity if there is no information about the geometry. For example, jets directed perpendicular to the LOS cannot be identified based only on line profiles. On the other hand, jets directed along the LOS may be spectroscopically, but not spatially, distinguished. One approach to study the different sources of an EE is to divide the line profile into its spectral components. High-resolution spectral and spatial observations, together with certain assumptions on the geometry of the events, would then allow us to distinguish the regions of moving plasma from those with plasma at rest, even if their projections are superimposed.

Estimates of the electron density as one of the most important plasma parameters have previously been made for the source regions of EEs, yielding values of $n_{\mathrm{e}} \approx 10^{10} \mathrm{~cm}^{-3}$ (Brueckner \& Bartoe 1983; Dere et al. 1991; Wilhelm et al. 1998; Pérez \& Doyle 2000). In this work, we attempt to determine the electron densities not only in the source regions of two EEs, but also in their environment. 


\section{Observations and data analysis}

The extreme-ultraviolet spectral range from $749 \AA$ to $789 \AA$ can simultaneously be observed with the solar ultraviolet measurements of emitted Radiation (SUMER) instrument on the Solar and Heliospheric Observatory (SOHO) using detector B in the first order of diffraction (Wilhelm et al. 1995). With the temporal resolution of SUMER, it is possible to devise observing schemes compatible with the time scales of short-lived phenomena, such as EEs. Many transition-region lines can be studied in this spectral range, including pairs of lines that allow an estimate of the electron density to be made (e.g., the O v $759 \AA / 761 \AA$ ratio).

Even though a slit spectrograph has many advantages for such studies, it lacks a high-resolution imaging capability. As such images are, in general, also not available from other instruments, certain EEs out of the many observed events have to be selected, for which geometrical assumptions can be made with some confidence.

The selected events were associated with the small active region NOAA7995 which crossed the centre of the disk on 17 November 1996. The radius of the Sun as seen from SOHO was $R_{\odot}=980^{\prime \prime}$, so that $1^{\prime \prime}$ corresponded to $710 \mathrm{~km}$ at that time. A $0.3^{\prime \prime} \times 120^{\prime \prime}$ slit was used with $1^{\prime \prime}$ resolution elements defined by the detector pixel size along the slit in the direction of the $y_{\mathrm{s}}$ axis, which is positive towards the north. The east-west resolution element was determined by the slit width and the telescope point-spread function of 0.6" (Lemaire et al. 1997). The integration time was $115 \mathrm{~s}$, which is rather long for EE observations, but allows us to telemeter the full spectral range for an analysis of all relevant emission lines to the ground. For each slit position and exposure, spectra are obtained for the 120 exposed spatial pixels of the detector. No raster scan of the slit was employed, but the solar rotation introduced an east-west drift of stationary solar features of $0.3^{\prime \prime} / 115 \mathrm{~s}$, which is effectively a slow scan motion of the slit image from west to east.

The first event, EE I, was observed around 03:20 UTC and the second one, EE II, around 10:00 UTC. Both took place near the centre of the solar disk $\left(x_{\mathrm{s}}=0\right.$; and $\left.y_{\mathrm{s}}=0\right)$. As a reference we present, in the left upper panel of Fig. 1, an image of the active region as seen by the $195 \AA$ filter of the extreme-ultraviolet imaging telescope (EIT) on SOHO (Delaboudinière et al. 1995) observed at 03:00 UTC, and a magnetogram (right upper panel) observed at 03:15 UTC by the Michelson Doppler Imager (MDI, Scherrer et al. 1995) on SOHO. In the lower panels, we show the corresponding maps for EE II. The dashed lines represent the slit projections corrected for the time differences as far as the solar rotation is concerned, and the arrows show the positions of the events. The first event occurred near the active region whereas EE II was observed inside the active region.

The events were selected because the distributions of the line shifts along the slit and also their temporal evolution allowed us to devise reasonable geometrical configurations. We denote the line shifts, in the usual way, as blue and red components, and interpret them as Doppler-shifted emission from ions moving towards and away from $\mathrm{SOHO}$ with respect to the reference. The reference (zero value in the Doppler-shift scale), was defined by the average of the wavelengths of the line over four exposures before and after the event. The components were separated from the core profiles by Gaussian fits. During EE I, the line profiles at many locations along the slit simultaneously increased in the blue and red wings. This can only happen if the activity occurrs either in a large area intersected by the slit or along a linear structure nearly aligned with the slit. For simplicity, we assume the latter possibility in the discussion below. On the other hand,
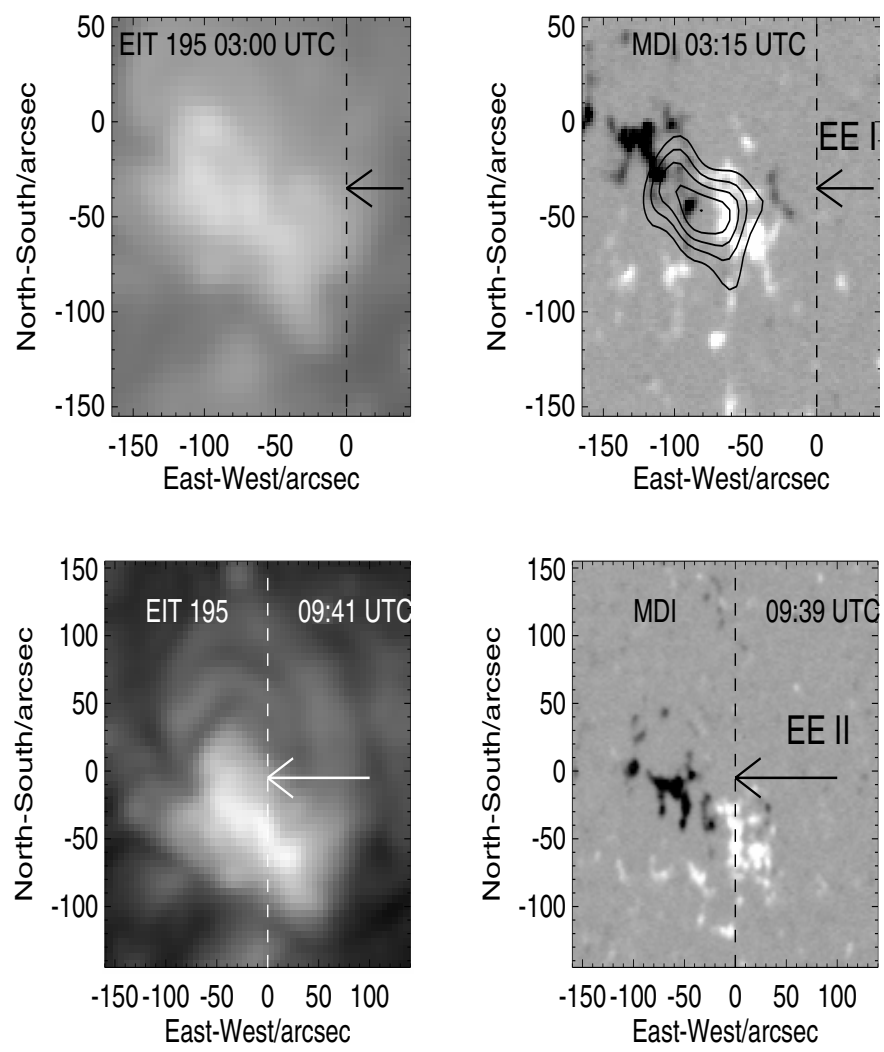

Fig. 1. Map of the active region NOAA7995 obtained in the $195 \AA$ wavelength band of EIT (Courtesy of the EIT consortium) on 17 November 1996 at 03:00 UTC (left upper panel), and the magnetogram observed on 17 November 1996 at 03:15 UTC (Courtesy of the MDI consortium). Over-plotted in contours, the strength of the radio emission is superimposed as observed by the Nobeyama Radioheliograph at 03:10 UTC (right upper panel). The second EIT map is taken at 09:41 UTC (left lower panel) and the MDI magnetogram at 09:39 UTC. In all the panels, the slit projection is shown by a vertical dashed line and the arrows point to the positions of EE I and II, respectively.

during EE II, the line profiles simultaneously increased at both wings only in a few locations. As the detector sees a distinct sequence as a function of the position of the slit with respect to the Sun, we will argue in favour of a small scale stationary event scanned by the spectrometer slit. The wavelength of the maximum of each fitted Gaussian was measured and the Doppler shift computed under the assumption that the undisturbed line was at the rest wavelength. Since we are observing Doppler velocities of more than $100 \mathrm{~km} \mathrm{~s}^{-1}$, such an assumption is accurate enough in this context.

Using the maximum of each Gaussian fit, we produced plots of the spatial distributions of the highest spectral radiances in the three spectral ranges. The distributions for EE I were made along the slit during the largest extent of the sources, whereas for EE II the profiles were measured at different slit positions during the sequence. In order to have an idea of the extent of the emissions, the spatial distributions of the radiances were measured as full width at half maximum (FWHM).

\section{Event I (EE I; 17 November, 03:20 UTC)}

This event occurred at $x_{\mathrm{s}}=0^{\prime \prime}, y_{\mathrm{s}}=-35^{\prime \prime}$. Its spectra are shown in Fig. 2. The time of each spectrum relative to the first frame at 03:16:07 UTC is given on the right-hand side of the figure. 


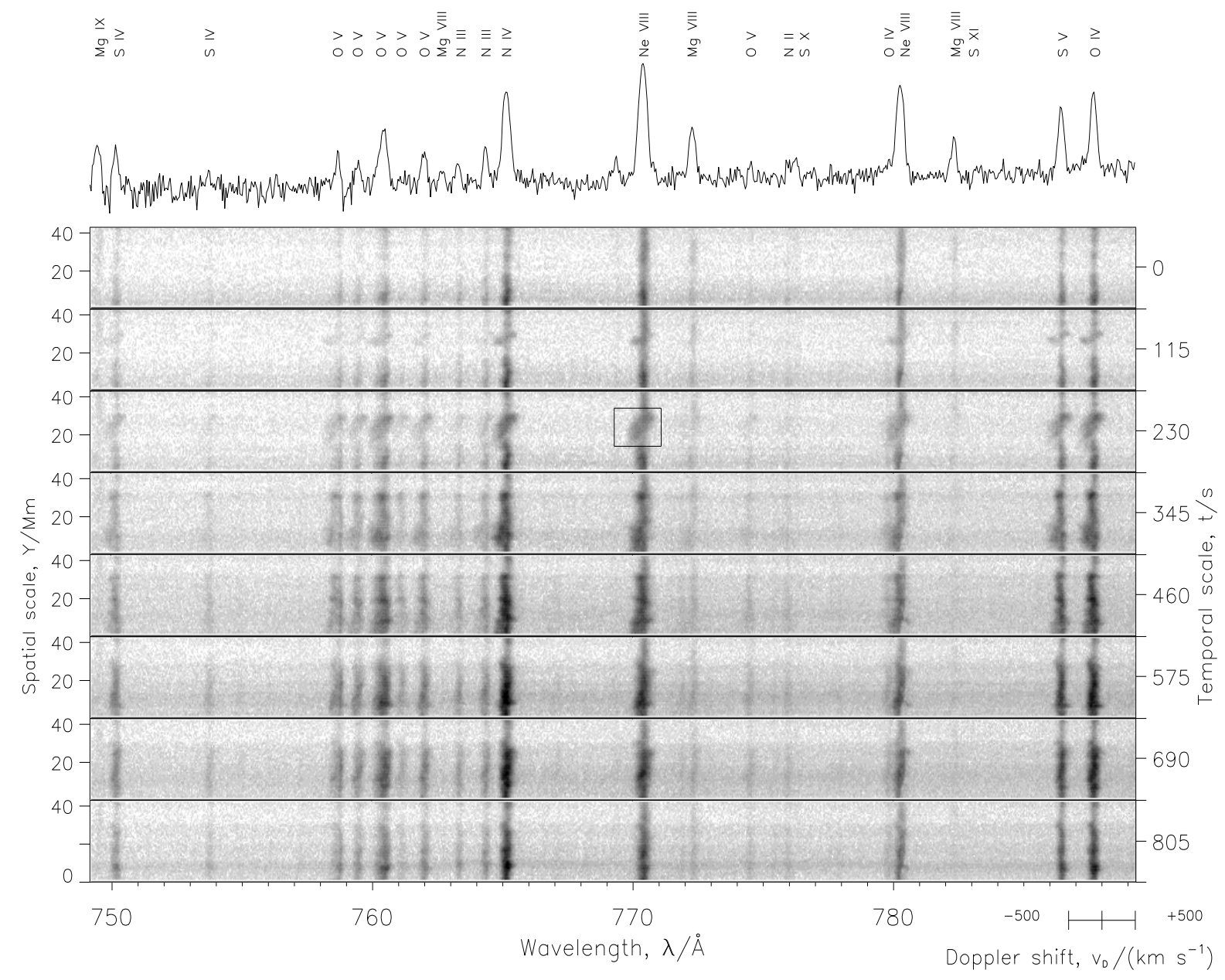

Fig. 2. Spectroscopic observations of EE I. Eight exposures of $115 \mathrm{~s}$ each, starting at 03:16:07 UTC, with a spatial coverage of $43 \mathrm{Mm}(60 \mathrm{px})$ are shown. The solar rotation introduced a motion of the slit image from frame to frame of $210 \mathrm{~km}\left(0.3^{\prime \prime}\right)$ with respect to the Sun. Red shifts are towards the right (the velocity scale is shown in the lower right-hand corner). North is down. A spectrum obtained prior to the event is shown as an amplitude plot at the top of the diagram with an identification of prominent lines. The O v lines at $759.44 \AA$ and $761.24 \AA$ formed at $2.4 \times 10^{5} \mathrm{~K}$ are a density-sensitive pair (Curdt et al. 1997). The event in the black rectangle around the Ne VIII line at $230 \mathrm{~s}$ is shown enlarged in Fig. 3.

\subsection{The EEI behaviour in different emission lines}

Blue and red spectral wings developed in the O IV, O v, S V, $\mathrm{N}$ IV, and Ne VIII lines near $Y=30 \mathrm{Mm}$ on the spatial scale of Fig. 2. The hot lines (Mg VIII and Mg IX) are barely visible and do not show any high-velocity signatures. These signatures are also weak for the cold N III lines. The onset of the event can be seen in the frame labelled $115 \mathrm{~s}$ as red and blue shifts slightly offset in north-south direction. In the next frame, the red and blue spectral distortions of the lines spatially widened to more than $15 \mathrm{Mm}$ in the north-south direction and looked similar in all prominent lines. In subsequent intervals the event subsided. Thereafter small EEs took place in the north. A conceptual geometry of the magnetic field configuration before and during the event is sketched in Fig. 4. The initial explosive event in Fig. 4a changes the magnetic field topology and is followed by a rearrangement of the field lines resulting in large scale blue shifts (sling effect) and a localized red shift (Fig. 4). See Shibata et al. (2007) for a detailed magnetic reconexion sketch.

The profiles of the Ne VIII $770 \AA$ line obtained for different locations of frame $230 \mathrm{~s}$ are shown in Fig. 3 in detail. The Ne VIII line is blue-shifted in the north, whereas it is red-shifted in the south.
In Figs. 5a and c, the radiance distributions of the bright lines N IV, and Ne VIII are shown for two exposures in Fig. 2. These curves were smoothed by averaging over three consecutive values obtained by the fits described in Sect. 2. The shapes of the $\mathrm{S} \mathrm{V}$ and O IV lines are similar. The corresponding Doppler shifts are shown in Figs. 5b and d. We note that for the analyzed lines, there is a spatial shift between the red and blue distributions.

Results pertaining to the strongest lines are listed in Table 1. $T_{\mathrm{F}}$ is the formation temperature of the lines, $s_{\mathrm{b}}$ is the FWHM of the distribution of the blue component along the spatial dimension, and $s_{\mathrm{r}}$ that of the red component. The event can be seen in $\mathrm{N}$ III and S IV $\left(T_{\mathrm{F}}=8 \times 10^{4} \mathrm{~K}\right)$ to Ne VIII. The extent of the blue component was about twice as long as that of the red one for all lines. The distributions were broader in the Ne VIII line than in the other lines. The red component attained its highest velocity of about $100 \mathrm{~km} \mathrm{~s}^{-1}$ around the location of the maximum of the red radiance distribution.

\subsection{The electron density during EE I}

The wings of the OV $759 \AA$ and $761 \AA$ lines $\left(T_{\mathrm{F}}=2.4 \times 10^{5} \mathrm{~K}\right)$ also increased during EE I. We separated the profiles into three spectral components to obtain estimates of the electron density 

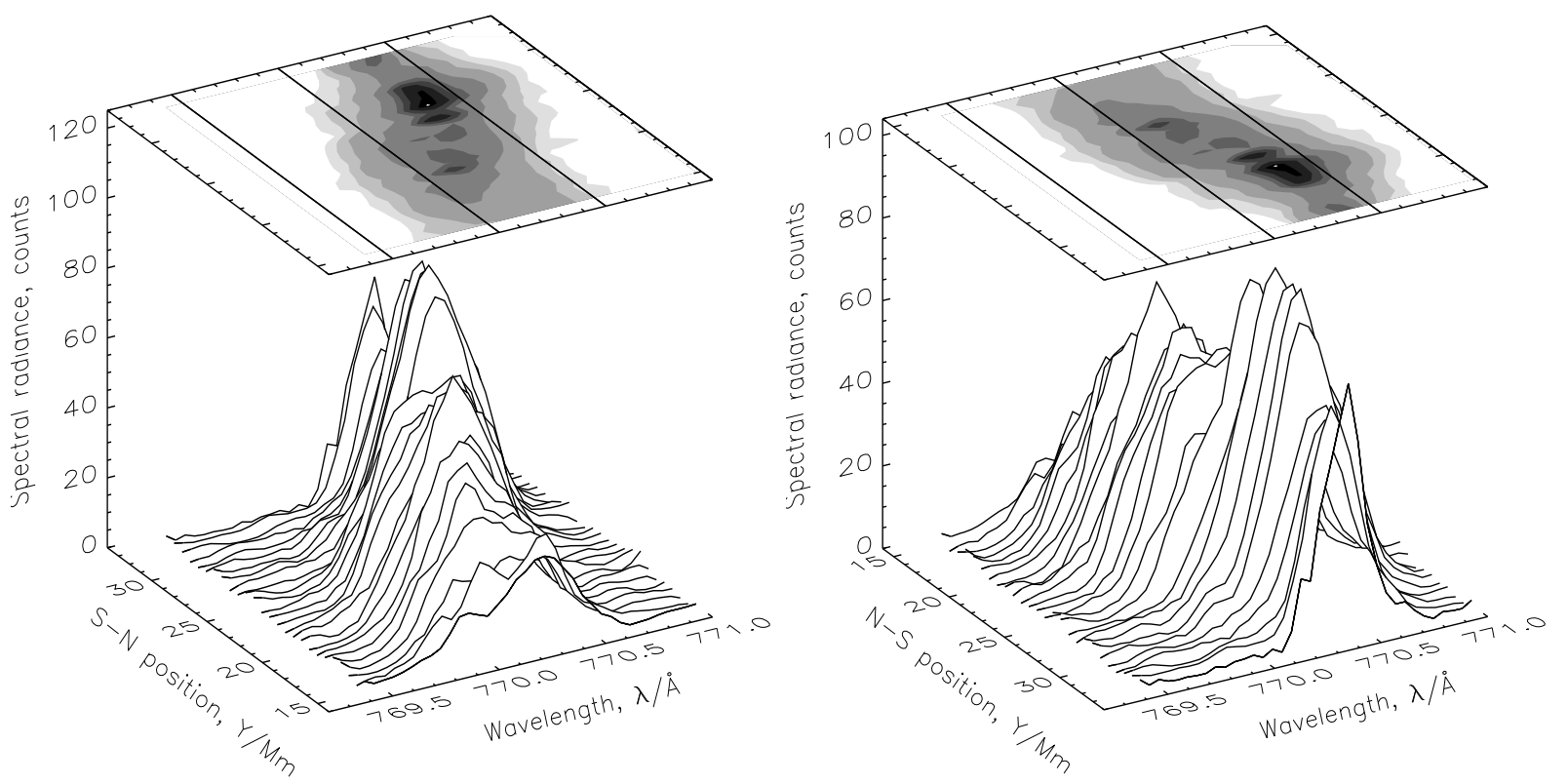

Fig. 3. The spectral profiles of the Ne VIII line at $770 \AA ̊$ observed during EE I at 230 s on our time scale (cf. Fig. 2). For clarity, we present two different views. In the left panel, profiles are seen from north to south, and from south to north in the right panel. On top of the panels the line radiance is shown in gray-scale representation.

at the locations of the core and the blue and red wings with the help of this density-sensitive line ratio (Curdt et al. 1997). The horizontal bars in Fig. 6 indicate the extent $(F W H M)$ of the distributions of the blue and red components. The density of the red region, $n_{\mathrm{e}}=\left(4.7 \pm 0.7 \times 10^{10}\right) \mathrm{cm}^{-3}$, was of the order of the density of the core $\left(4.3 \times 10^{10} \mathrm{~cm}^{-3}\right)$ but they were larger than that of the blue one $\left(9 \times 10^{9} \mathrm{~cm}^{-3}\right)$. The maxima of blue and core densities were located between the red and blue regions whereas the maximum of the electron density in the red component was offset to the south by a few megametres.

\section{Event II (EE II; 17 November, 10:00 UTC)}

This event was observed at $x_{\mathrm{s}}=0^{\prime \prime}, y_{\mathrm{s}}=-5^{\prime \prime}$. Its extent was only $\approx 2100 \mathrm{~km}$ (about $3^{\prime \prime}$ along the slit direction). The spectra over the whole range recorded are shown in Fig. 7 , and in Fig. 8. The Ne VIII $770 \AA$ line profiles are given at three consecutive time intervals. During the exposure prior to that shown in Fig. 8a, (in the second frame of Fig. 7), a spectral component appears on the red side of the Ne VIII line. It can be fitted by a single Gaussian, thus allowing us to express the observed curve as a sum of two Gaussians centred at different wavelengths and with different widths and amplitudes. In panel (a), the red component has grown, while a weak blue component appeared. In panel (b) the blue component is stronger than the red one. In the last frame (c), the red component is so weak that it does not appear in the image any longer, whereas the blue component remains very bright.

These observations may be interpreted either (1) as the result of scanning a stationary bipolar jet where the first portion seen was the red one. After that, both directions were detected, and in the last slit position only the blue jet was in the field of view, or; (2) as a temporal variation of the jet within the field of view (FOV).

Assuming similar dimensions in the direction along and perpendicular to the slit, a stable jet could indeed have crossed our FOV due to the solar rotation. This scenario would be similar to the one described by Innes et al. (1997). If, on the other hand, a)
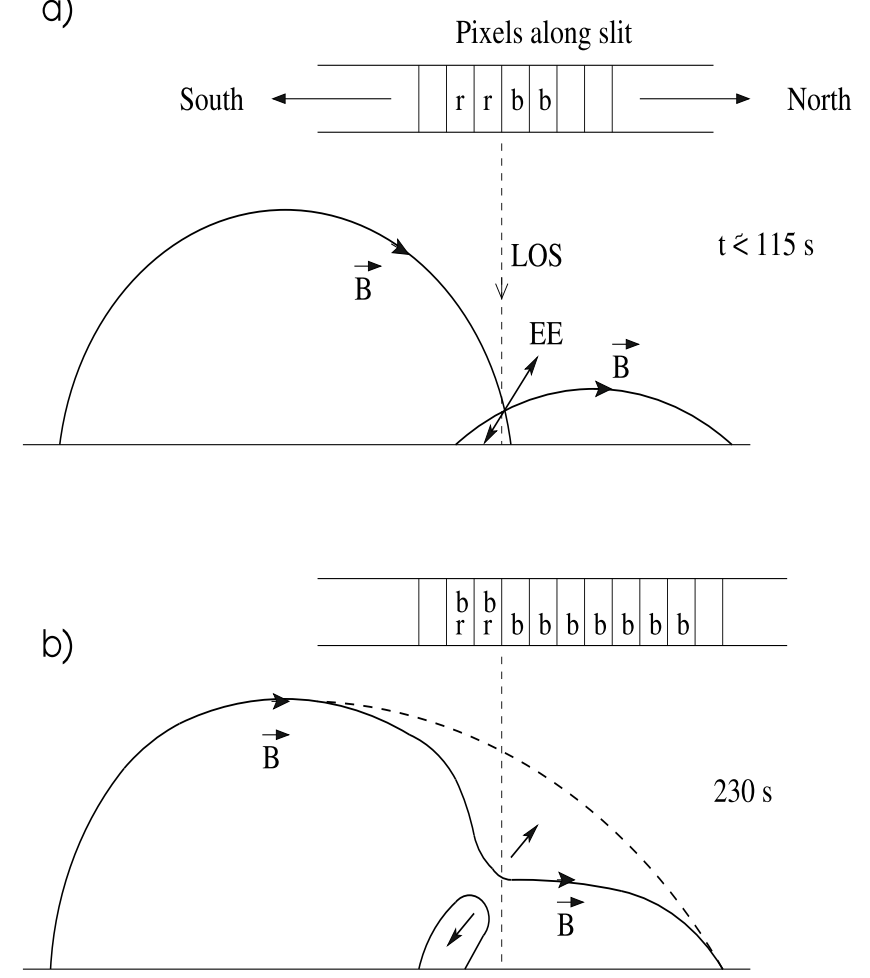

Fig. 4. Conceptual magnetic field configurations during EEI, a) at $115 \mathrm{~s}$ and b) at $230 \mathrm{~s}$. The direction of B is arbitrarily assumed as the magnetogram in Fig. 1 does not provide enough detail. $r$ and $b$ indicate red and blue shifts observed in a detector pixel along the slit.

we make the assumption of a temporal development, we have to explain the reversal of a $180 \mathrm{~km} \mathrm{~s}^{-1}$ jet from red to blue within 6 min inside an area of a few square arcseconds without changing the overall spectral characteristics in the immediate neighbourhood. Since the second assumption does not seem to be very likely, we will interpret this event as a spatial sequence in line 


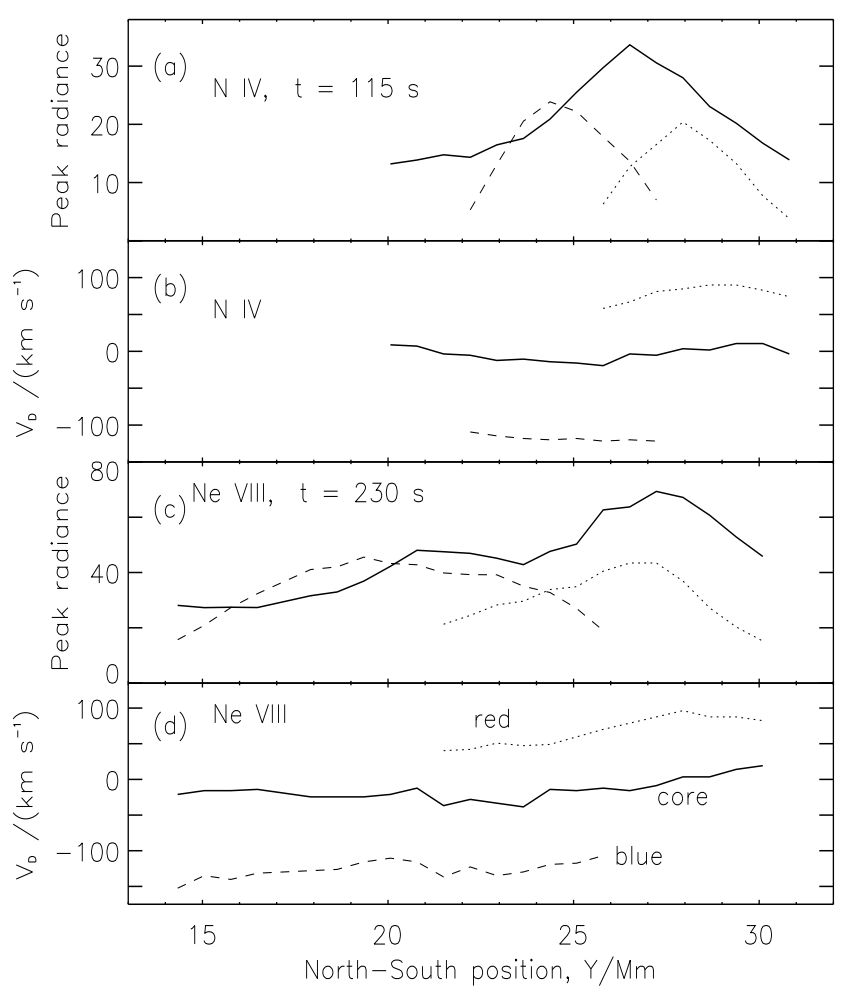

Fig. 5. Distributions of the spectral radiance components of the lines a) N IV $765 \AA$ at 115 s and c) Ne VIII $770 \AA$ at 230 s for EE I (north is to the left). The solid line represents the radiance (based on the maxima of the fitted Gaussians) of the core component, the dotted line shows the red and the dashed the blue components along the spatial dimension of the slit. The Doppler shifts of the three components of N IV and Ne VIII are shown relative to the position of the undisturbed lines in panels $\mathbf{b}$ ) and d).

with the statement in Sect. 2, but we can, of course, not rule out slow temporal variations. The geometry of this event and its observation is sketched in Fig. 9. It can then be concluded that the angular separation between the red and the blue jets is only about $0.6^{\prime \prime}$ in the scan direction.

This is in agreement with the Shibata et al. (2007) finding of a structure of a size less than $1^{\prime \prime}$ observed in white light. With both dimensions of the jet being very small, the angle between the jets and the LOS must consequently be small as well. We therefore expect that the radial velocities are not significantly different from the actual speeds, and a correction for LOS effects is not necessary.

\subsection{The EE II behaviour in different emission lines}

If, as we now have assumed, the jet is stable for at least a few minutes and nearly parallel to the LOS, the effects on the spectral characteristics of the neighbourhood should be symmetric with regard to the scan position, and indeed we see, upon close inspection of Fig. 7, very similar velocity shear features in S V, $\mathrm{O}$ IV and $\mathrm{OV}$ on either side of the bipolar jet. As these fade out with increasing separation from the jet, they are very likely related to it, and thus lend support to our assumptions. The velocity shears as such are extremely interesting.

All lines from N III to Ne VIII were affected by this event, although the cool lines do not have a pronounced red component. Since the LOS is more or less perpendicular to the solar surface, it appears as if the event manifests itself at all height levels

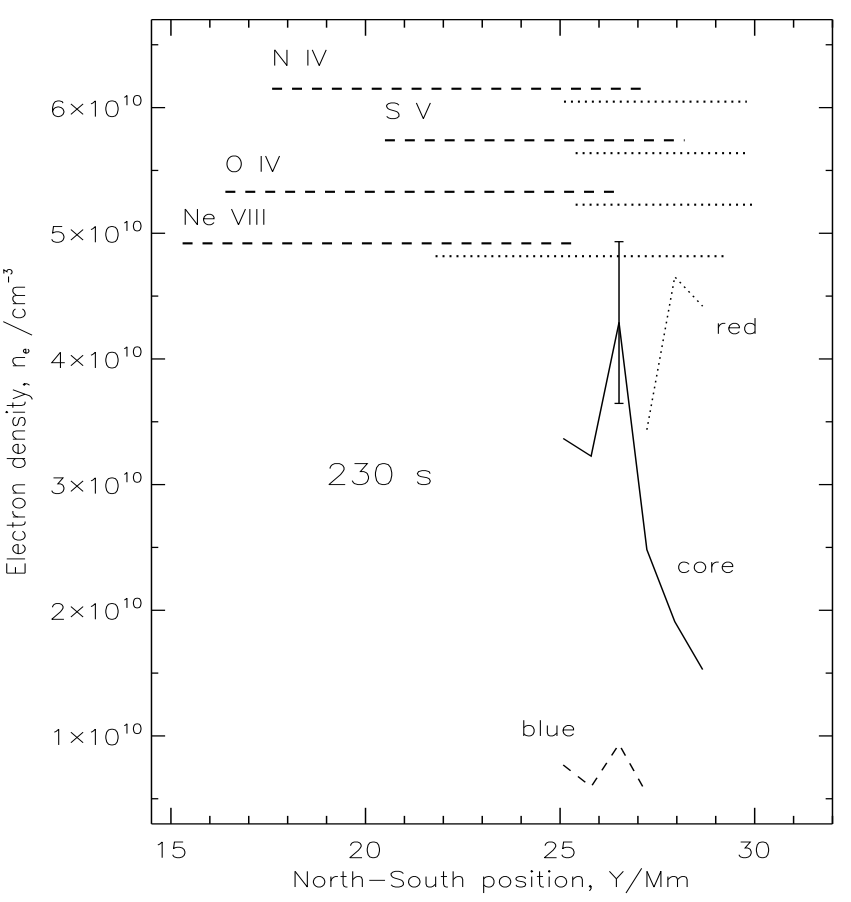

Fig. 6. Distributions of the electron density computed from the OV $759 \AA$ and $761 \AA$ line ratio during EE I. The solid line denotes the core, the dotted the red and the dashed the blue density distributions. The horizontal dotted lines show the extent $(F W H M)$ of the red and the dashed lines that of the blue components (cf. Table 1). It can be seen that the highest densities were attained between the two spatial radiance enhancements.

covered by the formation temperatures. It is bounded by the dense atmosphere at low altitudes.

As one would expect, all $\mathrm{OV}$ lines behave in a very similar fashion. There is, however, a marked difference in comparison to the Ne VIII lines: the $\mathrm{OV}$ red jet starts one time integration later with velocities of $+140 \mathrm{~km} \mathrm{~s}^{-1}$ to $+160 \mathrm{~km} \mathrm{~s}^{-1}$. This can be interpreted as an indication that the event started in the upper transition region near the formation temperature of the Ne VIII line $\left(6.2 \times 10^{5}\right)$.

The blue jet with velocities of $-70 \mathrm{~km} \mathrm{~s}^{-1}$ to $-90 \mathrm{~km} \mathrm{~s}^{-1}$ is very pronounced in the frame labelled $345 \mathrm{~s}$, when it actually dominates the line shape with a peak value three times higher than that of the undisturbed line.

The undisturbed O IV line at $788 \AA$ has a Doppler width of $35 \mathrm{~km} \mathrm{~s}^{-1}$. The peak radiance increases by a factor of two in the course of the event. At $230 \mathrm{~s}$ the red and blue wings of the line are clearly seen. The blue contribution at $345 \mathrm{~s}$ can be described as a significant bump with velocities of $-260 \mathrm{~km} \mathrm{~s}^{-1}$. The red contribution, although reaching up to $+280 \mathrm{~km} \mathrm{~s}^{-1}$ in velocity, is not very pronounced. It decreased and returned to relatively undisturbed conditions in the next frame, but with some smallscale Doppler shifts still present. The S V and N IV lines both behave very similarly, which is not surprising in view of their identical formation temperatures. They are also not very different from the hotter O IV line. S IV and N III show only a blue component from $230 \mathrm{~s}$ to $460 \mathrm{~s}$. The other lines, $\mathrm{Mg}$ VIII and S X in particular, are too weak for a determination of any details of their spectral shapes, but they all show weak radiance increases during this time interval.

We measured the radiance maxima and the Doppler shifts of the Gaussian profiles fitted to each line and performed the same analysis as for EE I, but as mentioned, in the direction of the 
Table 1. Dimensions of the blue $\left(\mathrm{s}_{b}\right)$ and red $\left(\mathrm{s}_{r}\right)$ components during the events EE I and II.

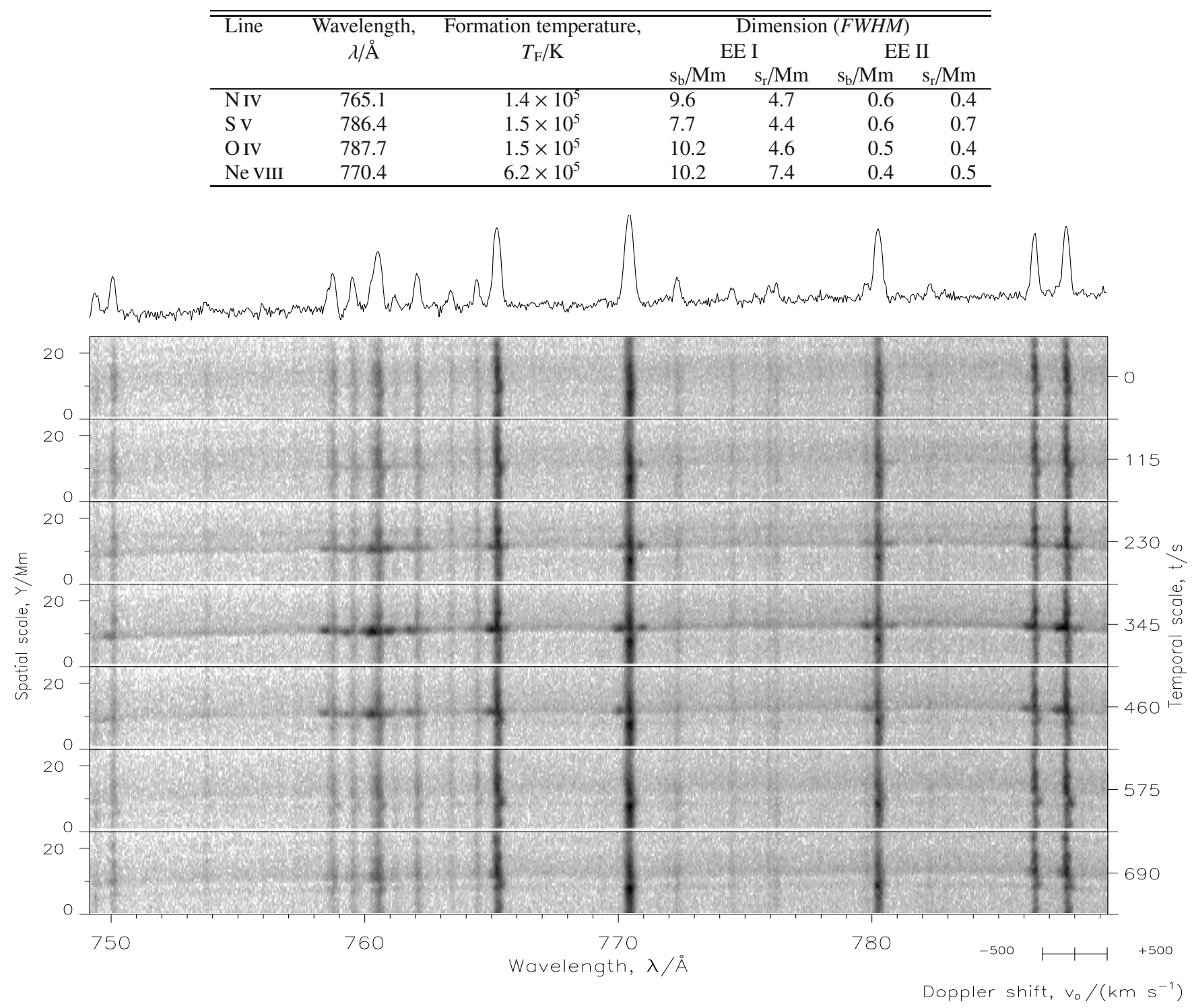

Fig. 7. Summary of the spectroscopic observations of EE II on 17 November 1996 at 10:00 UTC. The format is similar to that of Fig. 2. Seven frames are shown with spectra taken along a section of 36 spatial pixels. This corresponds to $25 \mathrm{Mm}$ on the Sun. The frames represent a temporal series with an exposure time of $115 \mathrm{~s}$ each, starting at 09:52:52 UTC and a total duration of $15 \mathrm{~min} 20 \mathrm{~s}$. The spatial variation of the discontinuities in the continuum result from uncorrected detector distortions.

slit image motion from west to east. The values obtained were averaged over three consecutive profiles of a given spectral component. The results for the Ne VIII line in Fig. 10 show that the radiance distribution of the red component was larger than that of the blue one. As seen from Table 1, the extent of blue and red jets, as a function of the formation temperature, was almost constant. This result could indicate that the width of the jets was constrained, probably in a magnetic flux tube. As for EEI, in EEII also there is a spatial shift between the red and blue radiance distributions.

\subsection{The electron density during EE II}

When the highest radiance values were attained during EE II, the blue wing was very strong (Fig. 7). Therefore, the O V lines at $759 \AA$ and $761 \AA$ became blended on the blue side, and it was not possible to determine the $\mathrm{O} V$ ratio. However, we could estimate the densities of the core and red components, the red being higher than $4 \times 10^{10} \mathrm{~cm}^{-3}$ and the core being higher than $1 \times 10^{10} \mathrm{~cm}^{-3}$ over a north-south extent of $2^{\prime \prime}$.

\section{Discussion and conclusions}

As already mentioned, the emission sites of EE I were rather large compared to those of EE II. The blue source of EE I was more extended than the red one. In contrast to this, the spatial extent of the red emission was larger than the blue one for EE II. Another difference was that the Doppler velocities of EE II were higher than those of EE I. We suggest that most of the differences result from a different perspective: the jet of EE II was directed more or less parallel to our LOS (cf. Fig. 9), whereas that of EE I formed a larger angle and initiated a magnetic field reconfiguration in the field of view of our slit (cf. Fig. 4). 

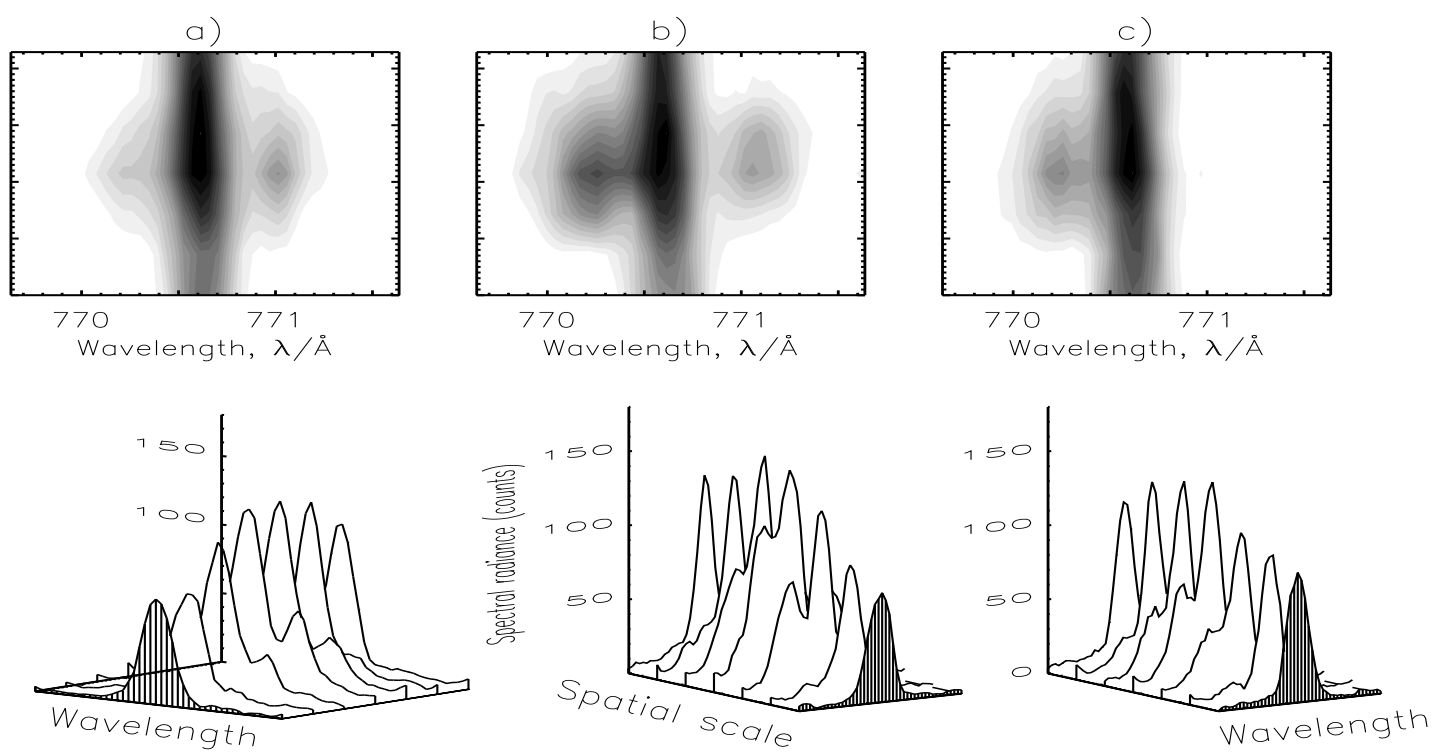

Fig. 8. a) Spectra of the Ne VIII $770 \AA$ A line taken on 17 November 1996 during EE II. The images show in gray scale the spectral radiance for a slit segment of six spatial pixels. North is up. The line profiles below are recorded for the seven different spatial locations of this segment. The first frame (on the left-hand side) was taken at 9:56:42 UTC and corresponds to the third frame (from top to bottom) of Fig. 7. The plots in b) and c) correspond to the two successive frames whose initial times are 9:58:37 and 10:00:32 UTC.

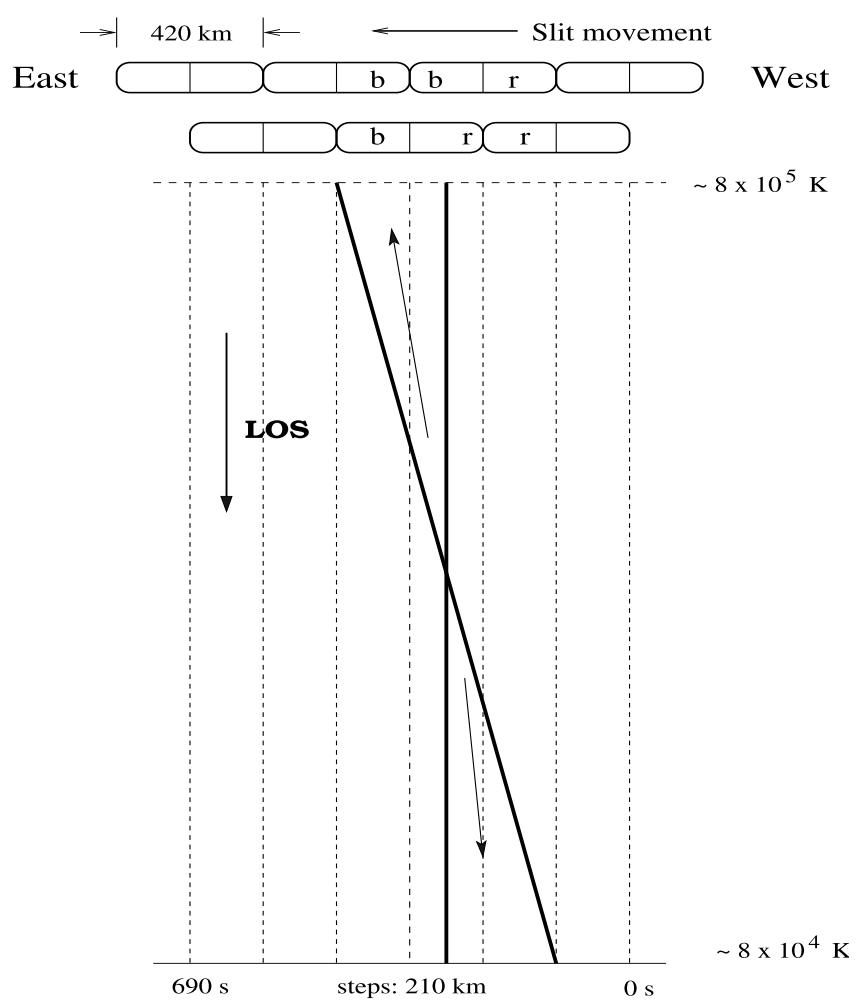

Fig. 9. Conceptual geometry of EEII. A stationary jet (more or less parallel to the LOS) is scanned in steps of $0.3^{\prime \prime}(210 \mathrm{~km})$ from West to East. Since the point-spread function is twice as large, the slit can cover red (r) and blue (b) shifts as indicated. The slit direction is perpendicular to the plane of the diagram.

The electron density enhancements of EE I are considerably narrower than the spatial radiance distributions. The density peaks between the red and the blue radiance maxima. This indicates that the radiance emission includes (at least in our projection) the region with the density enhancement, and is the result of processes related to the primary energy release. During EE II,

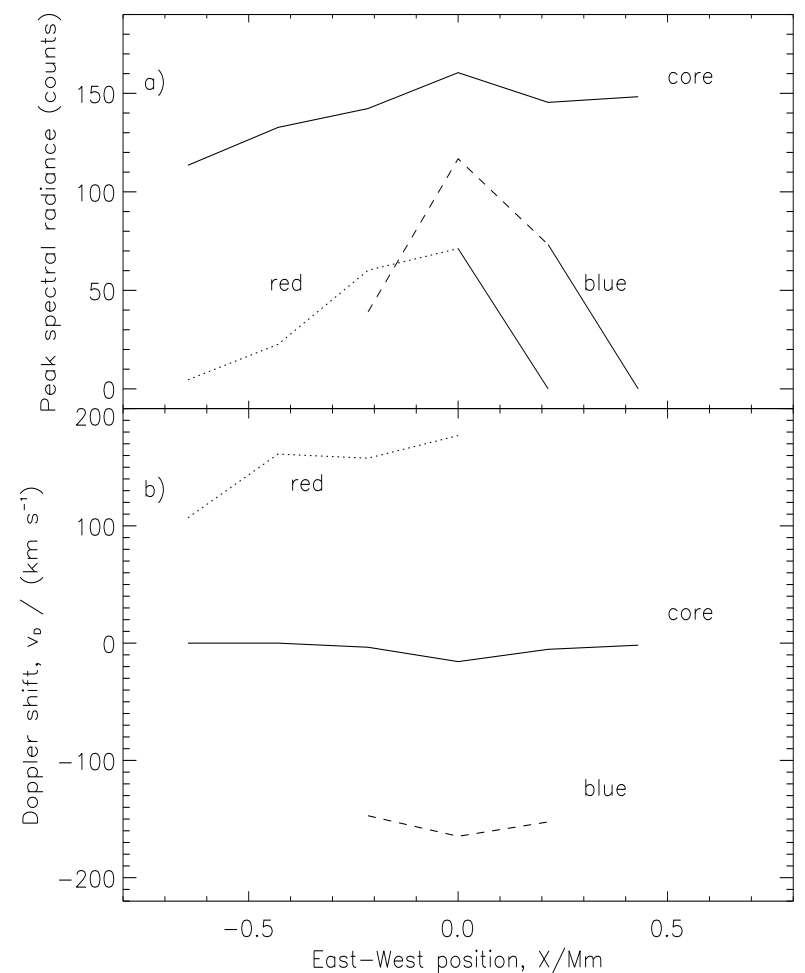

Fig. 10. a) Radiance and b) Doppler-shift distributions of the Ne VIII $770 \AA$ line for EE II. Dotted lines represent the values of the red component, while the dashed ones show those of the blue, and the solid line the core component.

the offset of the blue and red radiance distributions was considerably less than for EEI, which supports our assumption on the direction of the jet.

As seen in Fig. 5, the velocity distribution of EE I is completely different from the radiance distribution. However, when comparing these distributions, it may be seen that the radiance 
maxima of the red component are attained in the region where the plasma reaches the highest positive velocity.

The difference in the extents of the radiance enhancements and their variation with the formation temperature of the emission lines is clearly related to the orientation of the event with respect to the solar atmospheric density gradient. The maximum radiance did not occur at the primary source, as could be shown by the separation of the line profiles into the core and wing components.

From the analysis of the spatial distributions of the radiance and the electron density of the two EEs we conclude: (1) the shapes of the radiance distributions of EE I are similar from line to line, but are different for the three spectral components. The dimensions of EE II are much smaller than those of EE I (cf. Table 1), due to the different perspective; (2) the highest electron densities for the blue, core and red components of EE I occurred between the two radiance distributions. The sizes of the regions of increased density were considerably smaller than those of the radiance enhancements. The highest densities were observed for the red component.

Acknowledgements. The SUMER instrument and its operation are financed by the deutsches zentrum für luft- und raumfahrt (DLR), the centre national d'etudes spatiales (CNES), the national aeronautics and space administration (NASA), the european space agency's (ESA) PRODEX programme (Swiss contribution), and the max-planck-gesellschaft (MPG). The instrument is part of SOHO, ESA's and NASA's Solar and Heliospheric Observatory. We would like to thank D. Germerott for his help in data handling and calibration and also to T. Niembro-Hernández for her help in data reduction. E.M.-T. and A.L. acknowledge partial support from CONACyT (Grant No. 42577). A.L. acknowledges UNAM grant IN118906 and CONACyT grant 49395. We also would like to thank the anonymous referee for the useful comments and suggestions that allowed us to improve the paper.

\section{Appendix A: Example of Gaussian fittings}

The Gaussians are fitted to the observed line profile using a routine that applies the least-squares method. It gives the amplitude, the central velocity and the width for the fitted Gaussians. Then, the chi-square goodness-of-fit test is used as follows. It is defined the test statistic (Wall \& Jenkins 2003) as

$\chi_{0}^{2}=\sum_{i=1}^{n} \frac{\left(O_{i}-E_{i}\right)^{2}}{E_{i}}$

where $O_{i}$ is the $i$-value of the observed $n$ data points and $E_{i}$ is the $i$-value of the data set to be tested. If adjacent data points are combined into a bin, then $n$ is the number of bins. The value of $\chi_{0}{ }^{2}$ is compared with the values of the chi-square distribution $\left(\chi^{2}\right)$ for $v$ degrees of freedom (to be determined below). The aim is to identify the probability where $\chi_{0}^{2}$ equals $\chi^{2}$. This is the probability that the hypothesis is not rejected by the test.

The ratio $\chi_{0}^{2} / v$ is commonly used in tables and this value itself gives an idea ot the goodness of the fit. An hypothesis that leads to a $\chi_{0}^{2} / v$ considerably larger than unity or considerably smaller than unity has to be rejected (Bevington \& Robinson 2003).

In our case, an observed profile is a number of data points. The hypothesis is that the sum of $N$ Gaussians fits the observed data. For the analysis, low adjacent values are combined into bins. Therefore, in our test statistic $O_{i}$ is the $i$-bin of the line profile and $E_{i}$ is the result of the sum of $N$ Gaussians for that bin.

In the case of the chi-square goodness-of-fit test, $v$ is determined as $v=n-x-1$, where $n$ is the number of bins and $x$ is the number of variables. For a Gaussian curve we have

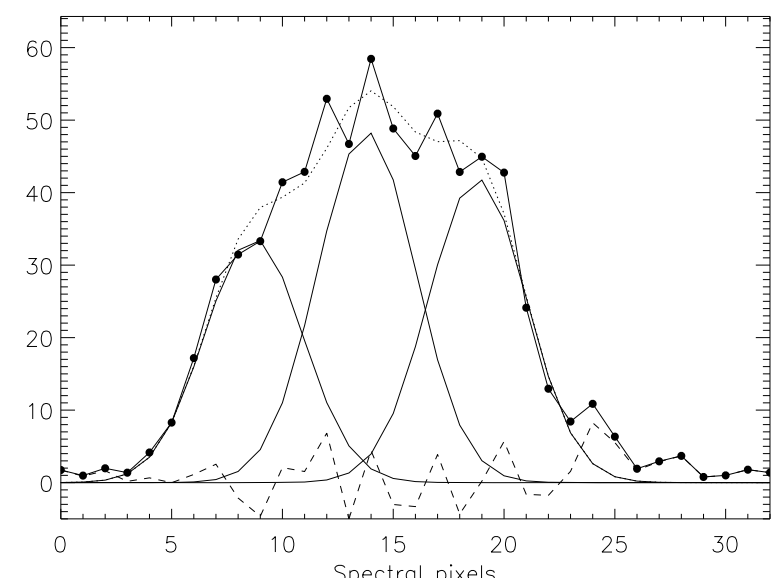

Fig. A.1. A Ne VIII line profile observed at $230 \mathrm{~s}$. The dots linked by a continous line denote the observed values and the three continous lines denote the Gaussians fitted by the least-square method. The dotted line is the sum of the Gaussians and the dashed line is the difference between this last curve and the observed profile.

three variables (amplitude, central velocity and width), then for $N$ Gaussians we would have $3 \times N$ variables and $v=n-3 * N-1$.

In Fig. A. 1 an observed profile is shown with three Gaussians fitted by the least-square method. Then, the hypothesis is that the three Gaussians fit the profile. The value of $\chi_{0}^{2}$ is 5.8 and $v$ is 9. Therefore, $\chi_{0}^{2} / v$ is 0.64 which, according to the chi-square distribution for nine degrees of freedom, corresponds to a probability of 0.76 . On the other hand, the hypothesis that two Gaussians fit the profile leads to $\chi_{0}^{2}=20$ which, for twelve degrees of freedom, corresponds to a probability smaller than 0.07 . Due to this low probability this last hypothesis is rejected and the hypothesis that three Gaussians fit the profile is adopted. If an hypothesis leads to a small probability then it is rejected.

\section{References}

Axford, W. I. 1985, Sol. Phys., 100, 575

Axford, W. I., \& McKenzie, J. F. 1992, in Solar Wind Seven, ed. E. Marsch, \& R. Schwenn, 1

Axford, W. I., \& McKenzie, J. F. 1997, in Cosmic Winds and the Heliosphere, ed. J. R. Jokipii, C. P. Sonett, \& M. S. Giampapa (Tucson: Arizona University Press), 31

Bevington, P. R., \& Robinson, D. K. 2003, Data Reduction and Error Analysis for the Physical Sciences, 68

Brueckner, G. E., \& Bartoe, J.-D. F. 1983, ApJ 272, 329

Chae, J., Wang, H., Lee, C., et al. 1998, ApJ, 504, L123

Curdt, W., Feldman, U., Laming, J. M., et al. 1997, A\&AS, 126, 281

Delaboudinière, J.-P., Artzner, G. E., Brunaud, J., et al. 1995, Sol. Phys. 162, 291 Dere, K. P., Bartoe, J.-D. F., Brueckner, G. E., et al. 1991, J. Geophys. Res., 96, 9399

Esser, R., Holzer, T. E., \& Leer, E. 1987, J. Geophys. Res., 92, 13377

Harrison, R. A., Harra, L. K., Brkovic, A., \& Parnell, C. E. 2003, A\&A, 409, 755

Innes, D. E., Inhester, B., Axford W. I., \& Wilhelm, K. 1997, Nature 386, 811

Landi, E., Mason, H. E., Lemaire, P., \& Landini, M., 2000, A\&A, 357, 743

Lemaire, P., Wilhelm, K., Curdt, W., et al. 1997, Sol. Phys. 170, 105

Mendoza-Torres, J. E., Torres-Papaqui, J. P., \& Wilhelm, K. 2005, A\&A, 431, 339

Pérez, M. E., \& Doyle, J. G. 2000, A\&A, 331, 334

Scherrer, P. H., Bogart, R. S., Bush, R. I., et al. 1995, Sol. Phys. 162, 129

Shibata, K., Nakamura, T., Matsumoto, T., et al. 2007, Sci., 318, 1591

Teriaca, L., Banerjee, D., Falchi, A., Doyle, J. G., \& Madjarska, M. S. 2004, A\&A, 427, 1065

Wall, J. V., \& Jenkins, C. R. 2003, Practical Statistics for Astronomers (Cambridge University Press), 124

Wilhelm, K., Curdt, W., Marsch, E., et al. 1995, Sol. Phys. 162, 189

Wilhelm, K., Innes, D. E., Curdt, W., et al. 1998, ESA SP-421, 103 